\title{
ACTUARIAL NOTATION
}

(To the Editors of the Journal of the Institute of Actuaries)

SIRS,

Mr M. E. Ogborn in paragraph 17 of his essay ( $\mathscr{I . I . A . ~ L x v I I , ~ p . ~ I 0 6 ) ~}$ suggests that if $\not{a}$ and $x$ be used for the present value and amount of an annuity-due (the symbol ${ }^{x}$ denoting payment in advance), then the symbol for discount should logically be $\stackrel{x}{\jmath}$, where $j$ is used to denote interest.

As the subject of acturarial notation is at present under consideration it may be of at least theoretical interest to record the simplification which would result if this unification of symbols were to be completed.

By analogy the force of interest and the force of discount should be represented by $\bar{j}$, so that in place of the series of equations connecting the existing compound interest symbols, viz.

$$
\left.\begin{array}{c}
\mathrm{I}-v^{n}=i a_{\bar{n} \mid}=j_{(m)} a_{\bar{n} \mid}^{(m)}=d \mathrm{a}_{\bar{n} \mid}=f_{(m)} \mathrm{a}_{\bar{n} \mid}^{(m)}=\delta \bar{a}_{\bar{n} \mid} \\
(\mathrm{I}+i)^{n}-\mathrm{I}=i s_{\bar{n} \mid}=j_{(m)} s_{\bar{n} \mid}^{(m)}=\ldots=\ldots=\delta \bar{s}_{\bar{n} \mid}
\end{array}\right\}
$$

we should have, writing $\mathrm{I}+i=w$ and $j$ for the rate of interest

$$
\begin{aligned}
& \mathbf{I}-v^{n}=j a_{\bar{n}]}=j^{(m)} a_{\bar{n}}^{(m)}=\jmath^{\times} \widehat{a}_{\bar{n} \mid}=\jmath^{(m)} \stackrel{\times}{n} \bar{n}^{(m)}=j \bar{a}_{\bar{n}]} \\
& \left.w^{n}-\mathrm{I}=j s_{\bar{n} \mid}=j^{(m)} s_{\bar{n} \mid}^{(m)}={ }^{\times \aleph_{\bar{n}}}={ }^{\times(m)}{ }^{(m)}=\bar{j} \bar{s}_{\bar{n}}\right\}
\end{aligned}
$$

Apart from a most desirable generality, such a system would have the merit of using only five basic symbols $j, v, w, a, s$ and three modifying symbols ${ }^{x},-,(m)$ instead of the nine basic symbols and two modifying symbols of the present notation.

If, in addition, $\mu$ were to be written $\bar{q}$ we should approach nearer to the ideal of using the bar for continuous functions and we should also leave the whole of the small Greek alphabet available for other purposes.

Incidentally, the above equations (B) give a further argument against the adoption of Dr van Haaften's proposal to write

$$
s_{\bar{n} !}=\frac{w^{n}-\mathrm{I}}{d}
$$

since the second line of (B) would then be

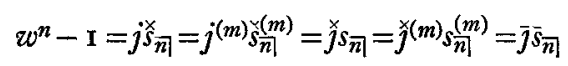

and the association of like symbols would be lost.

I am, Sirs, etc.,

M. H. GASTINEAU-HILLS

SYDNEY

18th December, 1936 